\title{
Bahía de Altata, Navolato - Nuevo modelo de desarrollo turístico sostenible
}

\section{Altata Bay, Navolato - New sustainable touristic model of development}

Martín León Santiesteban

Doctor en Gestión del Turismo. Docente de la Maestría de Gestión y Políticas Públicas de la Universidad de Occidente, Culiacán (México). ORCID: http:// orcid.org/0000-0002-8632-3833

martin.leon@udo.mx

Juan Pablo Mariño Jiménez

Doctor en Gestión del Turismo. Profesor Investigador de la Universidad Antonio Nariño, Bogotá

(Colombia).

jupamarino@gmail.com

Recepción: 26/07/2017. Aceptación: 03/03/2018. Publicación: 24/05/2018

Citación sugerida:

León Santisesteban, M. y Mariño Jiménez, J.P. (2018). Bahía de Altata, Navolato - Nuevo modelo de desarrollo turístico sostenible. 3C Empresa: investigación y pensamiento crítico, 7(2), 68-85. DOI: http://dx.doi. org/10.17993/3cemp.2018.070234.68-85/ 


\section{RESUMEN}

El presente capítulo permea el nivel de marginación de la población, la afluencia de visitantes y la fragilidad ecológica de los recursos naturales presentes dentro de la bahía de Altata y Navolato como determinantes para el desarrollo de nuevos proyectos con fines turístico - recreativos en la zona. Se encuentra pertinente evaluar la potencialidad de los recursos y atractivos existentes a la luz de potenciales atractivos turísticos sostenibles que permitan generar una diversificación económica sin elevar el deterioro ambiental que actualmente se está presentando. Finalmente, se dan a conocer los resultados de la aplicación de una metodología de evaluación cualitativa de los recursos de Altata, Navolato, encaminada a desarrollar un producto turístico de bajo impacto enfocado en actividades de turismo alternativo.

\section{ABSTRACT}

In this paper is easy to observe the level of marginalization of the population, the flow of visitors and the ecological fragility of this touristic area. It provides guidelines for developing new projects on the recreational-touristic use of resources with which it counts, which will generate economic diversification without raising the environmental deterioration that is currently taking place. In this way, it is necessary to make a comparison of the potential of existing resources and propose touristic sustainable products. The present document intends to present the results of the application of a methodology of qualitative evaluation of the resources of Altata, Navolato. In order to know its design as a low-impact touristic product focus on alternative touristic activities.

\section{PALABRAS CLAVE}

Altata, Recursos Naturales, Medio Ambiente, Sustentabilidad.

\section{KEY WORDS}

Altata, Natural resources, Environment, Sustainability. 


\section{INTRODUCCIÓN}

El objetivo del presente artículo es el de analizar los modelos de desarrollo para sentar las bases teóricas y metodológicas en la comprensión del fenómeno de masificación hacia la oferta de sol y playa, sus repercusiones en los recursos naturales, culturales y humanos, y su contraparte hacia el desarrollo de un turismo alternativo con prácticas sustentables y de bajo impacto.

\section{Se pretende evidenciar los resultados de la aplicación de una metodología de evaluación cualitativa de los recursos de Altata, Navolato, con el fin de conocer su diseño como un producto turístico.}

No es difícil observar el nivel de marginación de la población, la afluencia de visitantes y la fragilidad ecológica de esta zona turística, los cuales dan pauta para desarrollar nuevos proyectos sobre el uso recreativo-turístico de los recursos con los que cuenta, los cuales permitirán generar una diversificación económica sin elevar el deterioro ambiental por el que actualmente está pasando.

En el presente documento se pretende evidenciar los resultados de la aplicación de una metodología de evaluación cualitativa de los recursos de Altata, Navolato, con el fin de conocer su diseño como un producto turístico de bajo impacto enfocado a actividades de turismo alternativo.

Por lo tanto, se enmarca la definición de la sostenibilidad e incluye a esta Bahía para una mejor comprensión para su estudio futuro. Adicionalmente, se expone la metodología utilizada este documento, asimismo, se desarrolla un análisis sociodemográfico del puerto y se realiza la identificación del inventario turístico como potenciales a desarrollar en este lugar, tomando de referencia las propuestas realizadas por la Secretaria de Turismo (Sectur). Finalmente, se presentan las conclusiones del lugar en estudio la Bahía de Altata. 


\section{SOSTENIBILIDAD}

La sostenibilidad es un concepto cuyo origen se remonta a la década de los años ochenta del siglo pasado, el cual surge a partir de las diversas perspectivas científicas que para entonces discutían en torno a la relación existente entre el medio ambiente y las actividades económicas y productivas propias de la sociedad moderna (Medina, 2008).

Dentro de este contexto, Espino (2015), asegura que la sostenibilidad vincula todas aquellas necesidades económicas y biológicas con el propósito de estimar la magnitud del consumo humano, por lo que es un concepto ecléctico que se nutre de la economía, la sociedad y lo ambiental en procura de un bien común.

Organismos como Conservation of Nature and Natural Resources (IUCN), United Nations Environment Programme (UNEP) y World Wildlife Fund (WWF) (1980) refieren que la «sostenibilidad» surgió como resultado de analizar la situación del mundo en términos de afectación al medio ambiente, cuyo impacto en aquel entonces, constituía una situación insostenible que podía afectar el futuro de la humanidad.

En la Conferencia de las Naciones Unidas sobre el Medio Ambiente y Desarrollo de 1992, conocida como Cumbre de Río de Janeiro y se adopta la perspectiva planteada en la denominada Agenda 21, donde se declara cómo el desarrollo económico debe darse de manera paralela con el desarrollo social, en virtud a que las problemáticas de sobrexplotación del planeta están simbióticamente relacionadas con las realidades económicas y las disfunciones a nivel de justicia social (Guimarães, 2001).

Es por eso que, la explotación racional del medio ambiente, el trato consensuado con las comunidades locales y en general aquellos aspectos referidos por un gran número de autores a la hora de conceptualizar frente a la sostenibilidad, se enfocan mayoritariamente en las actividades industriales. Sin embargo, existen actividades que, como el turismo, tienen igual o mayor compromiso con las tres aristas de la sostenibilidad sin que dentro de su desarrollo medie un proceso fabril.

En este sentido, Ventura (2011), manifiesta que el turismo no es un producto, pero sí un complejo conjunto de servicios turísticos que se complementan con algunos bienes necesarios para la prestación de los mismos, cuya característica principal es la simultaneidad en la producción y el consumo geográficamente localizado en un espacio específico denominado destino turístico.

Es por tal razón que, ante la preeminencia de recursos de índole natural y cultural en los destinos 
turísticos Crouch (2007), enfatiza en que la sostenibilidad es el fundamento para conseguir factores de competitividad. Por lo que, investigadores como Diéguez, Gueimonde, Sinde, y Cerradelo (2011), destacan que la competitividad de los destinos turísticos puede incrementarse mediante una gestión basada en la calidad medioambiental y en el desarrollo de un turismo sostenible.

Para Ritchie y Crouch (2000), señalan que un destino turístico competitivo debe ser viable no únicamente en el plano económico sino también en lo ecológico, social, cultural y político, puesto que más allá de propender por una derrama económica producto de la explotación de los atractivos disponibles, la competitividad turística debe garantizar la sostenibilidad de los destinos.

Por lo tanto, promocionar los destinos bajo un criterio netamente comercial, conduce al abandono del principio de sostenibilidad (Ritchie y Crouch, 2003) y desconoce las implicaciones y los impactos que a nivel ambiental, cultural, económico y social trae consigo la actividad turística (Palma, 2007).

Al respecto, Diéguez, Gueimonde, Sinde, y Cerradelo (2011), (citando a Hassan, 2000), señalan que los destinos que consiguen tasas de crecimiento más elevadas son aquellos que establecen estrategias orientadas a la sostenibilidad medioambiental del turismo. No obstante Gómez (2011), crítica a la manera como la actividad turística ha sido asociada con una fórmula redentora de fácil consecución que pretende el logro de la competitividad a partir de los elementos medioambientales.

En este orden de ideas, según Ritchie y Crouch (2003), las ventajas competitivas o recursos desplegados son el resultado de la explotación sustentable de las ventajas comparativas a través del control, mantenimiento y mejoramiento de los recursos de un destino, dando cuenta de una gestión eficiente y eficaz en el largo plazo. De este modo, las ventajas comparativas de un destino turístico pueden evolucionar a ventajas competitivas dependiendo de la gestión que se desarrolle en procura potencializar su estructura, infraestructura y superestructura. 


\section{METODOLOGÍA}

Como fue señalado al principio, el objetivo del presente estudio es el evidenciar los resultados de la aplicación de una metodología de evaluación cualitativa de los recursos de Altata, Navolato, con el fin de conocer su diseño como un producto turístico de bajo impacto enfocado a actividades de turismo alternativo.

De esta manera, el enfoque cualitativo, para Cortes e Iglesias (2004), es una vía de investigar sin mediciones numéricas, tomando encuestas, entrevistas, descripciones, puntos de vista de los investigadores, reconstrucciones de los hechos, no tomando en general la prueba de hipótesis como algo necesario, y se llaman holísticos porque aprecian las cosas como un todo, sin reducirlos a sus partes.

En este sentido, se utilizó el método etnográfico basada en la observación, considerando recoger la información sobre las relaciones sociales y económicas de la población, para este caso se realiza un informe que incorpora la información recolectada y plasmada en una descripción integral de la comunidad en forma de monografía escrita (Angrosino, 2012). 


\section{SITUACIÓN SOCIO-DEMOGRÁFICA}

La Bahía de Altata se distribuye en 9 mil 100 hectáreas y se encuentra ubicado en el municipio de Navolato a 29 kilómetros de la cabecera municipal y a 60 kilómetros de Culiacán, capital del Estado de Sinaloa. Es posible arribar por la autopista a cuatro carriles Culiacán-Altata a través del uso del transporte urbano o bien en vehículo particular y en donde se pueden disfrutar de sus playas, pudiéndose practicar deportes acuáticos, la pesca, el motociclismo, el ciclismo y paseos en vehículos todo terreno (GES, 1990).

Igualmente, y a 5 kilómetros, del otro lado de la bahía, se encuentran las playas de El Tambor, donde se ha iniciado el desarrollo turístico de Nuevo Altata actualmente llamado Isla Cortes. Algunos de los factores que hacen particular a este sitio son el: el clima seco cálido con lluvias en verano y escasas a lo largo del año, lo registros indican que su temperatura oscila en promedio anual entre $\operatorname{los} 24^{\circ} \mathrm{C}(\mathrm{GES}, 1990)$.

\section{La Bahía de Altata tiene una afluencia constante de visitantes, principalmente los fines de semana, quienes llegan a disfrutar de sus atractivos y principalmente de su gastronomía a base de productos del mar.}

Su litoral se compone de una superficie baja y arenosa, donde sus mareas tienen características peculiares por ser de tipo mixto, es decir, con una combinación diurna de subida y bajada cada 24 horas. Esta Bahía se une a un extremo sur de la Isla de Redo y la punta del noroeste de la Península de la Lucernilla. Una de las principales actividades económicas de la Bahía de Altata es la captura de diversos productos marinos, entre los que destacan los de escama, moluscos y de los productos más importantes por el valor se encuentra el camarón dentro de la variedad de crustáceos.

Por último, la Bahía de Altata tiene una afluencia constante de visitantes, principalmente los fines de semana, quienes llegan a disfrutar de sus atractivos y principalmente de su gastronomía a base de productos del mar. Estas visitas a la playa se hacen por espacios cortos de tiempo de aproximadamente seis horas, ya que no cuenta con infraestructura hotelera. 


\section{OFERTA DE SERVICIOS EN ALTATA VS. ISLA CORTES (NUEVO ALTATA)}

La clasificación que se puede otorgar a este enclave turístico ubicado en la Bahía de Altata, en el municipio de Navolato, es para contextualizarlas, dependiendo de sus características, como zona turística, en este sentido se realiza una descripción considerando la elaboración de un sencillo inventario que integra la información descriptiva más relevante de los recursos de cada región, considerando que es un trabajo de valoración y de estructura de los productos que se ofertan en estos dos centros turísticos.

\subsection{ALTATA}

En este sitio turístico se observaron características preturísticas. De acuerdo con Molina (2007) se trata de ofertas y empresas que tienen bajo componente relativo de tecnologías y sistemas administrativos, el personal no requiere capacitación, no se promocionan, y registran instalaciones rudimentarias, siendo esto apreciado por los visitantes y pobladores, de ello, se da cuenta cuando concluye la carretera a cuatro carriles y pasa a circular en una calle normal de tierra a orilla de la playa.

Los restaurantes dan muestra del tipo de construcción en la diversidad de materiales utilizados, la mayoría cuenta con piso de cemento, sanitarios, cocina separada del área de los comensales, bardas bajas con vista a la playa, techos de palma en algunos casos y otros de cemento, cuenta con sistemas de abanicos, las mesas y sillas se observan en su mayoría estar elaboradas de madera y en pocos casos de plástico.

El personal que atiende al comensal en muchos de los casos corresponde a familiares dueños de los restaurantes y en algunos casos incorpora a niños a quienes se observa el ir y venir del restaurante al local con productos solicitados por los comensales. No se observan señalamientos respecto al estado que guarda las mareas, si bien es cierto que no existen olas en esta parte de la bahía, también es cierto la poca atención que se le presta a este tipo de señalamientos cuando las mareas son altas y se elevado el flujo de sus corrientes.

El cuidado al medio ambiente es claro que no existe un compromiso con la naturaleza en esta zona, pues al recorrer por la parte posterior del pueblo se encuentran grandes depósitos de basura cercanos a un manglar que conecta a un estero de la misma bahía. Se observa tala de árboles y mangles y, como consecuencia acarrea problemas de contaminación ambiental y visual, así mismo 
es un foco de infecciones para la misma población ya que se encuentra a escasos 300 metros de las viviendas.

Finalmente, en este apartado es importante señalar la falta de un muelle que pudiera considerarse exclusivo para las embarcaciones menores propiedad de pescadores, se observan dos, pero no cuenta con las condiciones para considerarse embarcadero, sino que son utilizados para aquellas unidades que realizan paseos a quienes visitan estas playas.

\subsection{ISLA CORTES (NUEVO ALTATA)}

En esta nueva infraestructura que se desarrolla se observan condiciones de un turismo industrial maduro, Molina (2006) explica que, en esta etapa, el turismo se instrumenta como una industria, es decir, un conjunto de empresas con diversos giros, que se guían por su código conceptual y operativo en el que predominan los principios de uniformización, centralización y maximización.

En el trayecto a la Isla Coretes, de oriente a poniente, la llegada a esta zona se realiza desviándose de la carretera de cuatro carriles a la derecha, con rumbo al campo pesquero de Dautillos, en el trayecto, kilómetro 6, se toma la desviación a la izquierda para topar con la entrada que da paso para cruzar el puente que conecta la bahía con la península. De esta manera, los visitantes pueden acceder al sitio cruzando un puente, considerado como emblemático por los lugareños, una vez en la parte más alta del mismo, la vista panorámica a ambos lados, se puede observar la belleza de la bahía a través del gran canal y del espeso manglar que lo adorna.

En el trayecto y al cruzar el puente se observan palmeras, las cuales fueron plantadas intencionalmente. Éstas no forman parte del tipo de vegetación propia de la región, adicionalmente en el trayecto la misma vía pasa a ser de cuatro carriles, es muy visible el espectacular que anuncia la llegada al sitio con su característico color azul y grandes letras blancas, se identifica en la parte baja del mismo, los logos de los sitios sociales, dando muestra de ser un lugar moderno.

Por lo que respecta al área de departamentos, estos se encuentran adornados con palma en sus techos y presumen de tener balcones construidos con maderos típicos de la sierra como es palo de Brasil y tablas de caoba o pino. Así mismo, se observa la construcción de un lago artificial, el cual agrega valor económico a este tipo de vivienda y, en el cual se permite realizar actividades acuáticas como son el cayac o pequeños botes impulsados por remos y no se permite circular con motor fuera de borda.

Se observa entre la vegetación unos techos de palma, los cuales se confunden con las ramas de 
mangle negro, acercándose se ubica un gran estacionamiento para los vehículos y da paso a la entrada al Club de Playa "Puesta del Sol", al ir ingresando pasa por un puente elevado de maderas bien talladas de forma circular, el cual se dirige a una pequeña cabaña que se observa al lado derecho y al izquierdo se percibe el ingreso a sanitarios y regaderas, separadas ambos para mujeres y hombres.

Por último, el cuidado de la naturaleza es claramente perceptible, la vegetación es abundante y no se observó destrucción de la misma, por otro lado, la recolección de la basura es realizada por la misma empresa que administra el lugar, de regreso se pudieron observar aves anidando en los árboles y fue posible observar codornices a orilla del camino. 


\section{RESULTADOS}

En la actualidad, el tema de la sustentabilidad ha venido cobrando relevancia, Molina (2006) explica que es en la etapa del turismo industrial donde los recursos existentes son los que determinan el tipo de turismo que se desarrolla. En este sentido, es de reconocer la importancia de una planificación integral, que contemple no sólo los aspectos económicos y sociales, sino que también los ambientales.

Para el caso de la Bahía de Altata la tarea requiere del esfuerzo conjunto entre los actores involucrados en el sistema turístico estatal y municipal, el que pueda ser aplicado en cualquiera de las modalidades o segmentos del turismo, llámese turismo tradicional o de naturaleza como se observa en el esquema, y el cual puede ser adoptado por la localidad.

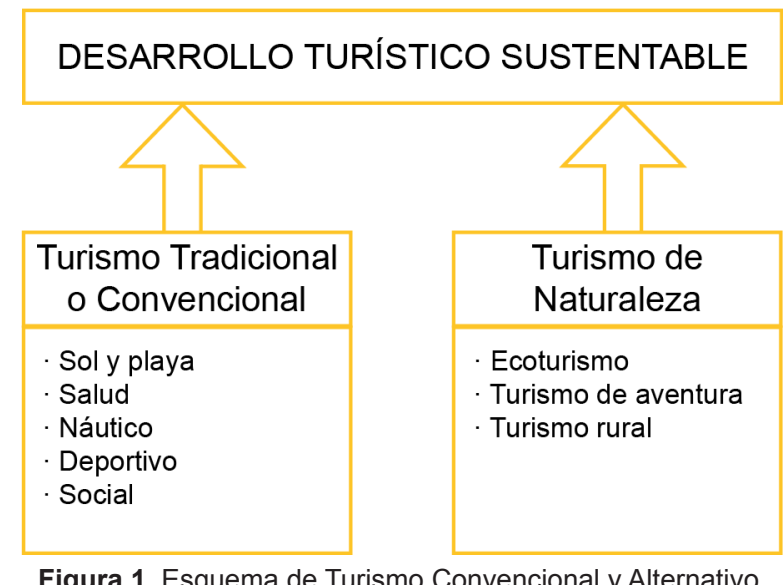

Fuente: Sectur (2004) Turismo Alternativo, Fascículo no. 1

Estas consideraciones permitirán plantear, basados en la definición que tiene la Sectur respecto al turismo alternativo: «Los viajes que tienen como fin realizar actividades recreativas en contacto directo con la naturaleza y las expresiones culturales que le envuelven con una actitud y compromiso de conocer, respetar, disfrutar y participar en la conservación de los recursos naturales y culturales» (Sectur, 2004) y que puedan ser implementadas en la Bahía de Altata, cada uno compuesto por diversas actividades, como las que a continuación se presentan:

- Ecoturismo: este tiene como fin realizar actividades recreativas de apreciación y conocimiento de la naturaleza a través del contacto con la misma (Sectur, 2004). 
- Observación de la naturaleza: En la selva se pueden observar la diversidad de plantas, como el cactus, la biznaga, papaches, choyas, flor de manzanilla y plantas medicinales como la cola de caballo y el ayal.

- Observación de la fauna: La visita a islotes, que sirven de anidamiento de aves marinas, en estos mismos se pueden observar pequeños mamíferos, en la selva existente, la paloma ala blanca, águilas y garzas, y también existen serpientes.

- Senderismo interpretativo: las dunas y grandes extensiones de playa sirven al visitante para transitar a pie o a caballo, equipado con señalamientos del tipo de flora, fauna y características del terreno.

- Talleres de educación ambiental: de las actividades antes mencionadas, se les puede incorporar actividades didácticas, pues en su mayoría involucran contacto directo con la naturaleza e involucrando a su vez a las comunidades locales.

- Turismo de aventura: Los viajes que tienen como fin el realizar actividades recreativas, asociadas a desafios impuestos por la naturaleza (Sectur, 2004).

1. Aire:

- Vuelo en globo: existen extensiones adecuadas para realizar esta actividad.

- Aeromodelismo: vuelo de pequeños aviones de control remoto.

- Vuelo en ultraligero: vuelo controlado en una pequeña aeronave con motor.

2. Tierra

- Caminata: las dunas y extensiones de playa sirven al visitante para transitar a pie, por caminos definidos y guiados por especialistas.

- Ciclismo de Montaña en dunas: las dunas y grandes extensiones de playa sirven al visitante para transitar en bicicleta.

- Cabalgata: las dunas y grandes extensiones de playa sirven al visitante para transitar a caballo, por camino a campo traviesa ya definido. 


\section{Agua}

- Buceo Libre: utilizando equipo básico como visor, aletas y snorkel se pueden observar entre el manglar a peces y cangrejos.

- Kayaquismo: utilización en esteros y bahía de una embarcación de diseño hidrodinámico, de una o dos plazas.

- Pesca recreativa: captura en mar abierto de peces como el dorado, marlín, atún, jureles y pez vela, bajo el concepto de capturar y soltar.

- Turismo rural: son los viajes que tienen como fin el realizar actividades de convivencia e interacción con una comunidad rural (Sectur, 2004).

- Agroturismo: existen terrenos destinados al cultivo y la ganadería rural, ocupado por una sociedad campesina lo que pudiera representar una alternativa económica a través de la combinación y el turismo.

- Talleres Gastronómicos: dar a conocer los platillos típicos de la región como la preparación de mariscos y pescados, barbacoas, tortillas de maíz y harina con grano molido, quesos, asaderas y pan.

En este sentido, el turismo alternativo que se presenta debe ser capaz de «promover un desarrollo integral para las comunidades, mitigando la pobreza (material) a través de la generación de empleo e ingresos económicos complementarios, evitando la migración de su población, fomentando una distribución justa de los ingresos y contribuyendo a la conservación del medio ambiente» (Inostrosa, 2008). 


\section{CONCLUSIONES}

La actividad turística para la Bahía de Altata debe ser entendido como parte del conjunto de actividades productivas que se llevan a cabo en las localidades pesqueras y campesinas, que ayude a ser un factor diversificador de la tradicional y dominante economía turística. Bajo esta lógica, el turismo no se puede convertir en la única fuente de rentas para las familias en la localidad.

La importancia del turismo alternativa radica en los cambios de tendencia donde es el propio turista quien está a la búsqueda de nuevas experiencias y únicas, acorde a sus gustos, necesidades y preferencias.

Aquí el objeto es el de satisfacer las necesidades actuales de las regiones anfitrionas, de los inversionistas turísticos, de los prestadores de servicios turísticos y de los turistas, con el fin de proteger, fortalecer y garantizar las oportunidades de desarrollo en el futuro.

Ante esto, la importancia radica en los cambios de tendencia donde es el propio turista quien está a la búsqueda de nuevas experiencias y únicas, acorde a sus gustos, necesidades y preferencias, como lo es el tener experiencias con las comunidades receptoras, la interrelación con la naturaleza, el de realizar actividades que le impliquen retos físicos, la búsqueda de emociones significativas, el mantenerse en forma, cuidar su salud y, el realizar actividades al aire libre. 


\section{REFERENCIAS BIBLIOGRÁFICAS}

Angrosino, M. (2012). Etnografia y observación participante en Investigación Cualitativa. Editorial Morata. Madrid, España.

Cortés Cortés, M. E. e Iglesias León, M. (2004). Generalidades sobre Metodología de la Investigación». Universidad Autónoma del Carmen, Campeche, México.

Grouch, Geoffrey I. (2007). Modelling destination competitiveness. A Survey and Analysis of the Impact of Competitiveness Attributes. Sustainable Tourism Pty. Queensland. Australia. p. 54.

Diéguez, I., Gueimonde, A., Sinde, A. y Cerradelo, L. (2011). Análisis de los principales modelos explicativos de la competitividad turística en el marco de la sostenibilidad. Cultur. p. 101-124.

Gobierno del Estado de Sinaloa. (1990). Navolato, monografia 1990, Ed. Gobierno del Estado de Sinaloa.

Guimarães, R. P. (2001). La sostenibilidad del desarrollo entre Rio-92 y Johannesburgo 2002: éramos felices y no sabíamos. Ambiente E̊ Sociedade, (9), 1 - 20.

Inostroza, Gabriel V. (2008). Aportes para un modelo de gestión sostenible del turismo comunitario en la región andina. Revista Gestión Turística, 10, Universidad Austral de Chile, 77-90.

IUCN, G., UNEP, U., y WWF, W. (1980). World Conservation Strategy Living Resource Conservation for Sustainable Development. Consultado: 02 de junio de 2016. Disponible en: https://portals.iucn. org/library/efiles/edocs/WCS-004.pdf

Maradiaga C., J. S. (1997). Perfil socioeconómico del Estado de Sinaloa y sus 18 municipios, Ed. UAS, 1a edición.

Medina, X. C. (2008). Caracterización de la industria forestal en España: aspectos económicos y ambientales. España: Fundacion BBVA.

Molina, S. (2006). El posturismo, turismo y posmodernidad. Trillas. México D.F. P. 109. 
Palma, G. (2007). Springbreakers: Turismo salvaje en playas mexicanas/Wild Tourism In Mexican Beaches. Grijalbo. P. 157.

Ritchie, J.R.B. y Grouch, G. I. (2000). The Competitive Destination. Tourism Management, 21, p. $1-7$.

Ritchie, J.R.B. y Grouch, G.I. (2003). The competitive destination: a sustainable tourism perspective. CABI Publishing. Cambridge, Reino Unido.

Secretaria de Turismo (Sectur). (2004). Turismo alternativo, una nueva forma de hacer turismo. Fascículo 1. Serie Turismo Alternativo. México, D.F.

Ventura, V. (2011). El turismo, su cadena productiva, y el desarrollo incluyente en América Latina: los casos de Brasil y México. Serie Comercio y crecimiento inclusivo, 138, 1-81. 


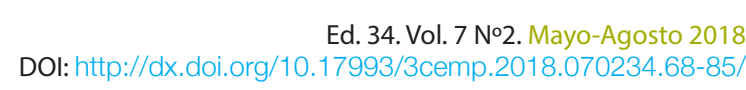

